\title{
Systematic Review and Patient-Level Meta- Analysis of SARS-CoV-2 Viral Dynamics to Model Response to Antiviral Therapies
}

\author{
Silke Gastine ${ }^{1, *}$, Juanita Pang ${ }^{2}$, Florencia A.T. Boshier ${ }^{2}$, Simon J. Carter ${ }^{1}$, Dagan O. Lonsdale ${ }^{3,4}$, \\ Mario Cortina-Borja ${ }^{5}$, Ivan F.N. Hung ${ }^{6}$, Judy Breuer ${ }^{2}$, Frank Kloprogge ${ }^{7}$ and Joseph F. Standing ${ }^{1}$
}

Severe acute respiratory syndrome-coronavirus 2 (SARS-CoV-2) viral loads change rapidly following symptom onset, so to assess antivirals it is important to understand the natural history and patient factors influencing this. We undertook an individual patient-level meta-analysis of SARS-CoV-2 viral dynamics in humans to describe viral dynamics and estimate the effects of antivirals used to date. This systematic review identified case reports, case series, and clinical trial data from publications between January 1, 2020, and May 31, 2020, following Preferred Reporting Items for Systematic Reviews and Meta-Analyses guidelines. A multivariable Cox proportional hazards (Cox-PH) regression model of time to viral clearance was fitted to respiratory and stool samples. A simplified four parameter nonlinear mixed-effects (NLME) model was fitted to viral load trajectories in all sampling sites and covariate modeling of respiratory viral dynamics was performed to quantify time-dependent drug effects. Patientlevel data from 645 individuals (age 1 month to 100 years) with 6,316 viral loads were extracted. Model-based simulations of viral load trajectories in samples from the upper and lower respiratory tract, stool, blood, urine, ocular secretions, and breast milk were generated. Cox-PH modeling showed longer time to viral clearance in older patients, men, and those with more severe disease. Remdesivir was associated with faster viral clearance (adjusted hazard ratio $(A H R)=9.19, P<0.001)$, as well as interferon, particularly when combined with ribavirin $(A H R=2.2$, $P=0.015 ;$ AHR $=6.04, P=0.006$ ). Combination therapy should be further investigated. A viral dynamic dataset and NLME model for designing and analyzing antiviral trials has been established.

Finding antivirals that target severe acute respiratory syndromecoronavirus 2 (SARS-CoV-2) will be crucial in managing the ongoing pandemic. In addition to the development of novel agents, substantial efforts are underway to establish whether currently available agents may be repurposed. ${ }^{1}$ A key biomarker for clinical antiviral activity is viral load in bodily fluids and assessing a drug's or drug combination's ability to reduce viral load is an important first step in identifying therapies that influence clinical outcome.

To correctly assess antiviral activity, it is first necessary to understand viral load natural history. As a rapidly progressing, primarily respiratory viral infection, SARS-CoV-2 elimination from the body seems to be mainly driven by a combination of innate immune response and exhaustion of target cells available for infection. ${ }^{2}$ Observational cohort studies published to date have shown that the rate of viral load decline seems slower in older patients, those with more severe disease, and those with comorbidities, such as diabetes mellitus and immunosuppression. ${ }^{3-6}$ Interpreting these observational studies requires caution because patients have often received antiviral therapies. Due to the timepoint of initial infection being unknown, assessing viral load in response to treatment must account for time since symptom onset. ${ }^{7}$

Since February 2020, case reports and case series of patient-level viral dynamics have been published, some of which report dosing of antiviral drugs. ${ }^{8}$ Clinical trials of antivirals and their association with viral load are also beginning to read out. ${ }^{9}$ Meanwhile, large pragmatic trials of repurposed monotherapy antivirals have yet to find a clearly effective agent. ${ }^{10}$ At this crucial juncture, it is vital to develop a pharmacodynamic modeling framework that can be used to describe the natural history of SARS-CoV-2 viral dynamics, make initial estimates on antiviral efficacy of agents used to date, and to design and evaluate phase II trials using viral load as a biomarker.

This systematic review therefore aimed to search for case reports, case series, and clinical trials reporting serial individual patientlevel SARS-CoV-2 viral load measurements in humans from any sampling site upon which an individual patient-level meta-analysis was then performed. A nonlinear mixed-effects (NLME) viral dynamic model was fitted to describe the viral trajectories in each sampling site and to give a quantitative measure of viral dynamics. In data of sufficient quality, the parameters of multivariable Cox

\footnotetext{
${ }^{1}$ Infection, Immunity and Inflammation Research and Teaching Department, Great Ormond Street Institute of Child Health, University College London, London, UK; ${ }^{2}$ Division of Infection and Immunity, University College London, London, UK; ${ }^{3}$ Department of Clinical Pharmacology, St George's University of London, London, UK; ${ }^{4}$ Department of Intensive Care, St George's University Hospitals NHS Foundation Trust, London, UK; ${ }^{5}$ Population, Policy and Practice Research and Teaching Department, Great Ormond Street Institute of Child Health, University College London, London, UK; ${ }^{6}$ Division of Infectious Diseases, Department of Medicine, The University of Hong Kong, Hong Kong, China; ${ }^{7}$ Institute for Global Health, University College London, London, UK. *Correspondence: Silke Gastine (s.gastine@ucl.ac.uk)
}

Received November 20, 2020; accepted February 22, 2021. doi:10.1002/cpt.2223 
proportional hazards (Cox-PH) regression models of time to viral clearance, and NLME models of antiviral efficacy were estimated.

\section{METHODS}

\section{Protocol and registration}

The protocol for this systematic review and individual patient metaanalysis, which follows the Preferred Reporting Items for Systematic Reviews and Meta-Analyses (PRISMA) Individual Patient Data systematic reviews guideline, ${ }^{11}$ was first published on May 27, 2020, at: https://github.com/ucl-pharmacometrics/SARS-CoV-2-viral-dynam ic-meta-analysis. The final dataset and statistical analysis code are also published here. The review was registered with PROSPERO (CRD42020189000).

\section{Eligibility criteria}

This study aimed to identify serial viral loads with time in human subjects infected with SARS-CoV-2 in order to describe and model viral load trajectory. The inclusion criteria were therefore papers containing individual subject-level reports of viral load with time, either since symptom onset or time since start of monitoring for asymptomatic subjects, and sampling site. Authors of manuscripts describing summary statistics of viral load with time were contacted requesting participant level data. Viral load was defined as either a value in copies $/ \mathrm{mL}$ or a cycle threshold $(\mathrm{Ct})$ value of an uncalibrated polymerase chain reaction (PCR) assay.

\section{Overall search strategy}

Since SARS-CoV-2 was notified to the World Health Organization (WHO) on December 31, 2019, we did not expect to find relevant papers published prior to this date. Hence, PubMed, EMBASE, medRxiv, and bioRxiv were searched with a date range of January 1, 2020, to May 31, 2020. The following search terms were used for PubMed and EMBASE: SARS-CoV-2 OR COVID OR coronavirus OR 2019-nCoV AND viral load OR cycle threshold OR rtPCR OR real-time PCR OR viral kinetics OR viral dynamics OR shedding OR detection OR clinical trial. Due to character limits in the search engine, the following search terms were used for medRxiv and bioRxiv: SARS-CoV-2 OR COVID-19 OR coronavirus AND viral load OR cycle threshold OR PCR OR viral dynamics OR clinical trial.

After removing duplicates, two reviewers independently identified papers for full text screening, with any discrepancies resolved by a third reviewer.

\section{Data extraction}

Viral loads were reported as either numerical values in tables, figures, or in viral load vs. time plots. Where possible, numerical values were copypasted directly into a comma-separated value format from the source, whereas tabulated numerical values contained in pdf images were extracted using https://extracttable.com/. Viral loads reported in plots were extracted using Web Plot Digitizer. ${ }^{12}$

Each viral load was paired with a time since symptom onset, or in asymptomatic subjects, the time since viral monitoring started. Furthermore, sampling site and, if viral load not reported in copies/mL, the PCR assay, including the primers used, were extracted along with limit of quantification and limit of detection, if available. The following patient-level covariates were extracted if available:

- Presence of fever $>37.5^{\circ} \mathrm{C}$ at any time (non-time-varying covariate)

- Age, where possible individual age but otherwise the study's reported central measure (e.g., mean and median)

- Sex or the male/female ratio was extracted if patient-level data not reported
- Need for and days of intensive care treatment

- Need for and days of mechanical ventilation

- Whether the patient died and time to death from symptom onset.

In addition, a standardized disease score was constructed for each patient as follows:

0 - asymptomatic

1 - mild disease (fever, cough, or other mild symptoms reported)

2 - moderate disease (in addition to mild criterion: need for supplemental oxygen/noninvasive ventilation)

3 - severe disease (requirement for mechanical ventilation).

All data were stored on a shared github repository, and standardized Rscripts took data from each paper to merge into a single master dataset. A quality control check on viral load values and all covariates was performed for each paper by an independent reviewer.

\section{Data quality assessment}

Viral load quality score. Two quality assessments were applied to each dataset. First, the quality of viral load reporting was rated on a 1-3 scale. The highest quality 1 was assigned to studies reporting viral load in copies/mL or reporting a calibration curve allowing for direct conversion of Ct values to viral load. Quality 2 was assigned if viral load was reported in PCR Ct and primers used in the assay were reported, but calibration data was missing. In this case, a published calibration curve for that primer from another source was used to convert to viral load in copies/mL. ${ }^{13,14}$ Where more than one calibration curve was available for the same primer the mean slope and intercept was used. The lowest (quality score 3) was assigned when viral load was reported in PCR Ct but no further information was available on the PCR assay. In this instance, a conversion to copies $/ \mathrm{mL}$ was made using the mean slope and intercepts from all calibration curves.

Drug quality score. The second quality assessment on a three-point scale related to reporting of the antiviral drug therapy administered: which drug(s) and upon which days did patients receive the $\operatorname{drug}(\mathrm{s})$. The highest quality 1 was assigned when it was reported which days each patient received each drug, or these data were provided by corresponding authors. If it was reported that no antiviral was administered, this was also assigned quality 1 . Quality 2 was assigned when antiviral drug treatment was reported, but ascertaining which days the patient had received the drugs was not possible. The lowest category, quality 3 , was assigned when it was not possible to determine whether or not antivirals had been administered.

\section{Statistical analysis}

Primary analysis of time to viral clearance using Cox proportional hazards modeling. The primary analysis was conducted on observed time to viral clearance, which was analyzed fitting Cox-PH regression models with adjusted hazard ratios (AHRs) estimated for each covariate. We verified the assumptions of proportional hazards using the Therneau-Grambsch test. ${ }^{15}$ The data used for this analysis were limited to respiratory and stool sampling sites only, as virus was found to be mostly undetectable at other sites. Furthermore, only data from patients with known antiviral history (drug quality 1 and 2) were used. To assess the possible risk of bias in different drug and viral load qualities, the analysis was repeated on two further subsets: first, with only drug quality 1 and respiratory samples, and second on assay quality 1 data only.

Time to viral load dropping below the limit of detection (LOD) was modeled with Cox-PH regression in $\mathrm{R}$ (version 3.6.3). ${ }^{16}$ Where a single 
patient contributed samples from multiple sampling sites (e.g., upper respiratory and stool), the time to the last site testing negative was used. Multivariable models for covariate effects on time to viral clearance were fitted, with additional interaction terms for drug therapies included, where multiple antiviral agents were given simultaneously. In studies reporting sex as a proportion of male patients, 10,000 datasets were simulated using the reported fraction of male patients to randomly assign individuals to being male patients from the binomial distribution. The Cox-PH regression model was then fitted to each dataset and parameter estimates compared with the model, where individual sex was assigned by rounding the fraction of male patients. Model parameter estimates were visualized using Forest plots.

Secondary analysis antiviral pharmacology model. The secondary analysis was to use an NLME model to quantify the increase in viral elimination rate with antiviral therapy. This analysis used data only from respiratory samples and rated drug quality 1 .

Nonlinear mixed-effects viral dynamic model. First, a descriptive analysis of all data was undertaken. An NLME viral dynamics model was fitted to the individual patient-level viral load vs. time data. The structural model was based on the general target cell limited model, which has previously been used to describe respiratory viral infections. ${ }^{7,17}$ This model consists of three ordinary differential equations relating to changes in uninfected target cells $(T)$, infected target cells $(I)$ and free virus $(V)$ over time $(t)$, as follows:

$$
\begin{aligned}
& \frac{d T(t)}{d t}=-\beta T(t) V(t) . \\
& \frac{d I(t)}{d t}=\beta T(t) V(t)-\delta I(t) . \\
& \frac{d V(t)}{d t}=\rho I(t)-c V(t) .
\end{aligned}
$$

where $\beta$ is the rate at which target cells become infected in the presence of virus, $\delta$ is the death rate of infected cells, $\rho$ is the rate of viral production from infected cells, and $c$ is the rate of clearance of free virus. This model is structurally unidentifiable, as tested through the "IdentifiabiltyAnalysis" package in Wolfram Mathematica version 12.1 (Wolfram Research, Champaign, IL), ${ }^{18}$ unless the initial condition for $T, \beta$, or $\rho$ are known. Furthermore, the elimination rate of free virus $(c)$ is likely to be much faster than the death rate of infected cells $(\delta)$. Hence, by assuming a quasi-steady-state between $I$ and $V$, and normalizing the total cell number by the number of infected cells when observations begin $(t=0)$, it is then possible to reduce the model to a structurally identifiable, two-state ordinary differential equation model relating to the fraction $(f)$ of infected cells with time and infected cells as a proxy for viral load as follows ${ }^{19}$ :

$$
\begin{gathered}
\frac{d f(t)}{d t}=-\beta f(t) V(t) . \\
\frac{d V(t)}{d t}=\gamma f(t) V(t)-\delta V(t) .
\end{gathered}
$$

with $\gamma$, a new parameter equal to $\rho \beta T_{\delta} / c$ and interpreted to be the maximum rate of viral replication. $\delta$ can now be interpreted as overall viral elimination rate. This population model was then fitted to viral load data with time using the following form:

$$
y_{i j}=f\left(\varphi_{i}, t_{i j}\right)+\varepsilon_{i j}
$$

where $y_{i j}$ was the viral load from subject $i$ at time $t_{i j}, f$ is the nonlinear model defined above with parameters $\varphi_{i}$, and $\varepsilon_{i j}$ the residual between the model prediction and the observed data.

Four parameters were estimated: the initial viral load at symptom onset $\left(V_{0}\right), \beta, \delta$, and $\gamma$. Interindividual variability was estimated for $V_{0}, \beta$, and $\delta$ with each assumed to follow a log-normal distribution. Viral loads were $\log$ transformed and the residual error was assumed to follow a normal distribution. Parameter estimation by maximum likelihood was undertaken using the stochastic approximation expectation maximization in NONMEM version 7.4. ${ }^{20}$ Model evaluation was undertaken by analysis of normalized prediction distribution errors and visual predictive checks. ${ }^{21}$ Viral loads below the LOD were included by integrating the density function from minus infinity to the LOD to yield a probability of the data being below the LOD ("M3 Method"). ${ }^{22}$

In some participants, multiple samples were taken at the same timepoint (either different sampling site or the same sample assayed by more than one method). In this case, a common residual error term was used to allow for modeling one-level nested random effects.

Descriptive analysis of viral shedding by sample site. The above model was fitted to data from each sampling site. The resulting parameters were then used to simulate the overall population viral load trajectories. For the respiratory sample sites viral area under the curve (AUC), peak viral load and half-life were derived from the model and plotted vs. patient covariates.

Covariate analysis and antiviral drug effects modeling. The initial model used only data obtained in untreated patients. A covariate analysis was undertaken testing the influence of sampling site (nasal vs. oral vs. lower respiratory tract), sex, age, and disease status on $V_{0}, \beta$, or $\delta$. Covariates were retained in the model based on the likelihood ratio test with a threshold level of significance of $P<0.01$, and if the same covariate addition to $V_{0}, \beta$, or $\delta$ all gave significant improvement to model fit then the model with the largest decrease in -2 log likelihood (NONMEM objective function value $(\mathrm{OFV}))$ was chosen. For the final model viral AUC, peak viral load and halflife were derived and plotted vs. patient covariates.

Using the final demographic model, data from patients undergoing antiviral treatment (antiviral drug quality 1 ) were added. A univariable analysis was performed, testing each drug's ability to increase $\delta$. Drugs showing significant improvement in model fit $(P<0.01)$, according to the likelihood ratio test, were then included in the final multivariable model.

\section{Simulations based on the antiviral pharmacology model}

Simulations were performed to explore the change in viral trajectories for different timepoints of therapy initiation: day 1 after symptom onset, day 3 , day 7 , and day 10 . Interferon and ribavirin monotherapy along with the combination therapy interferon plus ribavirin were explored this way. A dummy population of 5,100 subjects with ages uniformly distributed across 50 to 100 years, consisting of an equal ratio of men and women was created. Each regimen was simulated using the entire population, assuming sampling from the upper respiratory tract or nose for a time window of 14 days. Comparisons of the sample size required to detect a significant difference in the proportion of undetectable virus between antiviral and no treatments were made after 7 days of treatment with a $90 \%$ power and alpha level of $P<0.05$ for antivirals starting at days 1,3 , and 7 post-symptom onset.

\section{RESULTS}

Results of the systematic search are given in Figure 1, and details of included papers in Table 1. Individual patient-level data were 


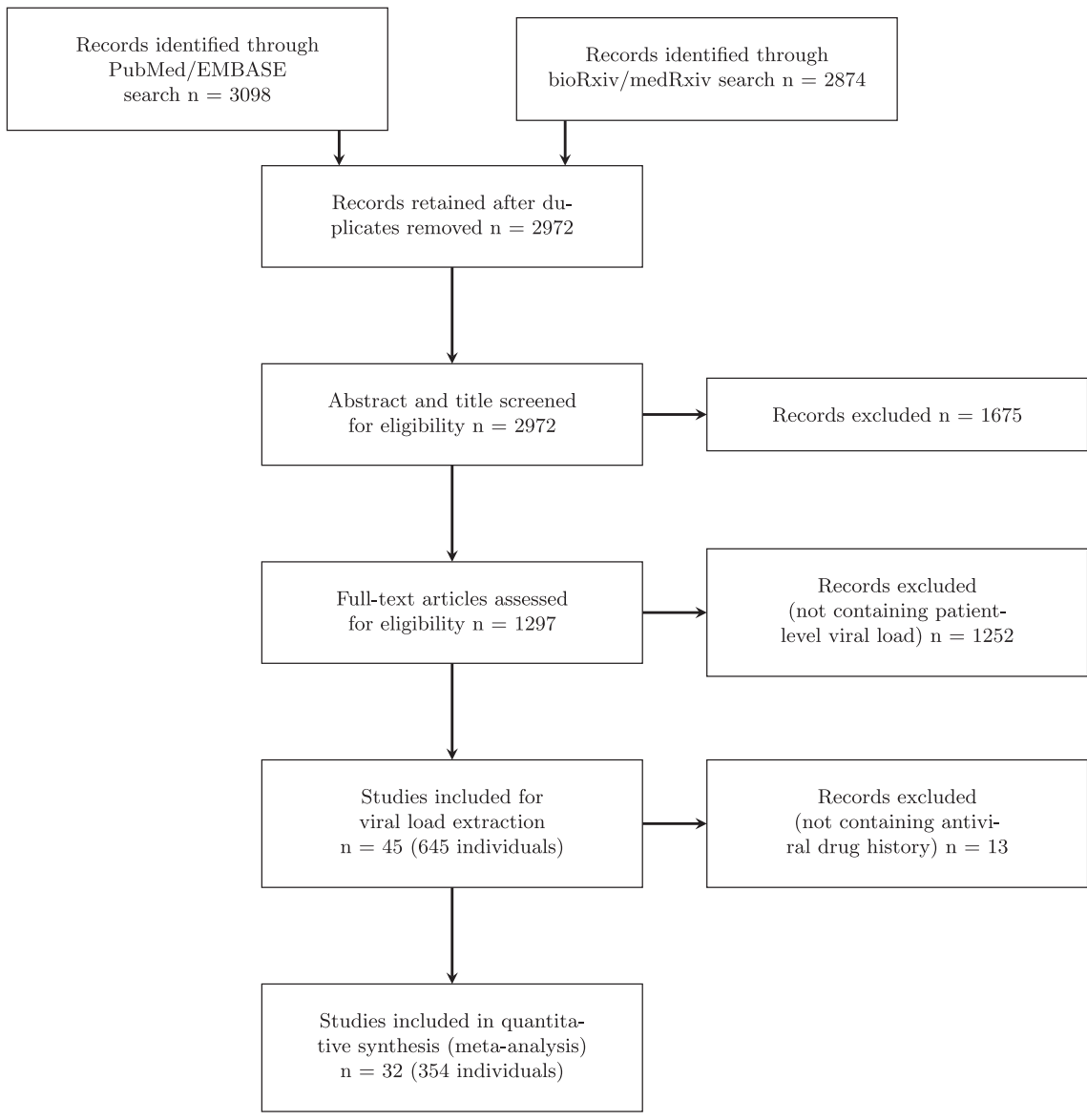

Figure 1 Preferred Reporting Items for Systematic Reviews and Meta-Analyses (PRISMA) diagram detailing the systematic search results.

extracted from 45 articles reporting viral loads and/or PCR Ct values with time since symptom onset. Of these 32 papers, either reported antiviral participant-level drug histories or these were provided by the corresponding author. The full dataset contained 645 individuals contributing 6,316 viral load samples. The majority of samples $(n)$ were taken from the respiratory tract: nasopharyngeal (315 individuals, $n=2,208)$, oropharyngeal or saliva (381 individuals, $n=2,144$ ), and lower respiratory tract (81 individuals, $n=799$ ). The other reported samples sites were stool/rectal swabs (99 individuals, $n=655$ ), blood/plasma (42 individuals, $n=258)$, urine (31 individuals, $n=112$ ), ocular (16 individuals, $n=50$ ), and breastmilk ( 4 individuals, $n=90$ ). Metrics of the full dataset are given in Table $\mathbf{S 1}$.

Full details of the extracted patient-level covariates are given in Table 2. Recording of fever, days in the intensive care unit, and days ventilated was largely unavailable. Therefore, no further analysis was performed on these variables. However, it was possible to categorize disease status in all drug quality 1 and 2 papers, either through reports in the manuscript or by contacting corresponding authors. Overall, most patients had mild disease $376(66.8 \%)$, whereas $79(14.0 \%)$ patients had moderate and 84 (14.9\%) had severe disease. In total, 24 (4.3\%) asymptomatic patients were reported. The distribution of recorded drug therapies, available for drug quality 1 data and respiratory site samples, is summarized in Table S2. Sixty-seven of these patients did not receive antivirals.

The NLME model fits to the overall data, stratified by sampling site, are provided in Table $\mathbf{S} 3$ and Figure $\mathbf{S 1}$. Simulations from the models for each sampling site showing the expected viral load trajectory along with the predicted proportion of samples, that would be below the LOD are given in Figure 2. For respiratory sites, model-derived AUC, peak viral load, and half-life are given in Figure S2.

Data on a total of 354 patients with respiratory and/or stool/ rectal sampling and drug quality 1 or 2 were available. A Forest plot of the parameter estimates from the Cox-PH regression model is provided in Figure 3. Viral clearance was fastest from upper respiratory tract samples and slowest from stool. More sensitive assays (with lower detection limits) were associated with longer time to viral clearance and viral clearance was faster in female patientss, younger patients, and those who were asymptomatic.

Regarding antiviral therapies, only remdesivir $(\mathrm{AHR}=9.19$, $P<0.001)$ and interferons (AHR $=2.20, P=0.015)$ were independently associated with faster viral clearance. The effect of interferon alpha and beta (Figure S3) was similar and hence these were combined. Lopinavir/ritonavir, ribavirin, and interferons were most used and most used in combination. Adding interaction terms for interferon plus lopinavir/ritonavir, interferon plus 
离

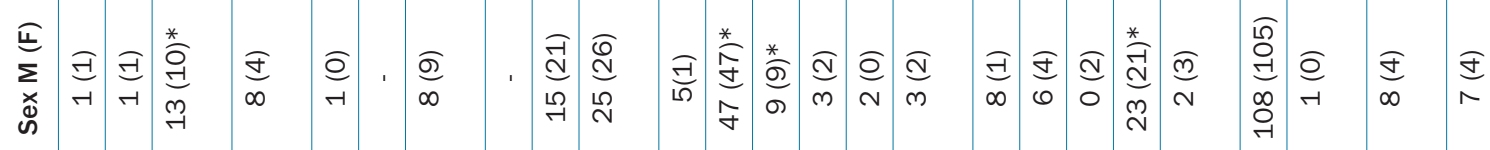

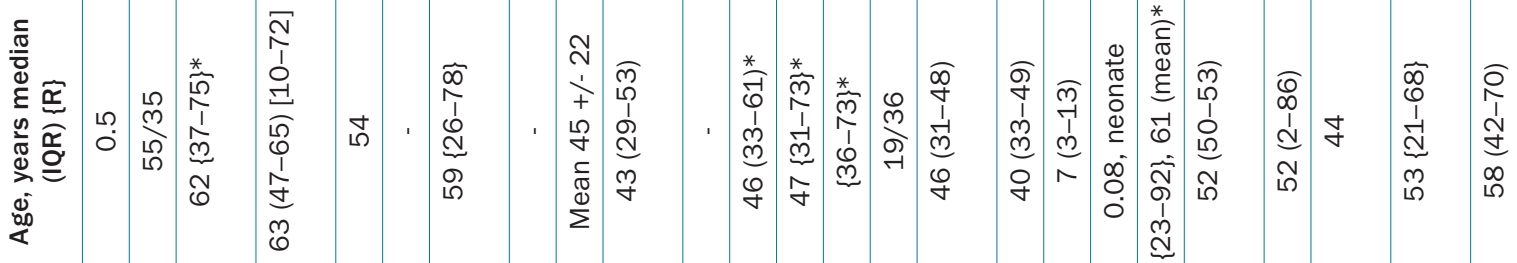

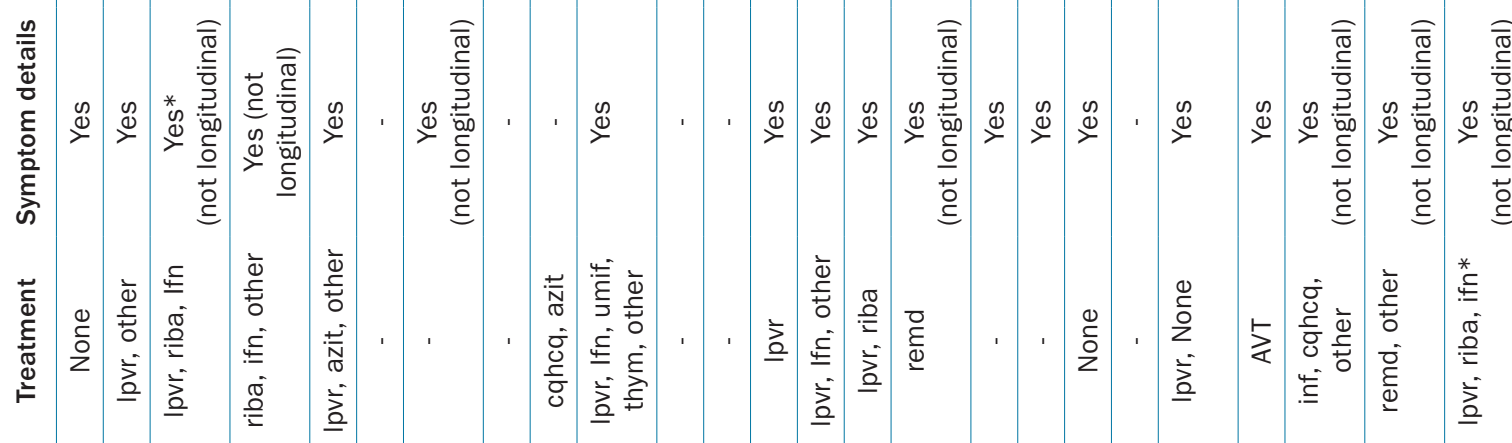

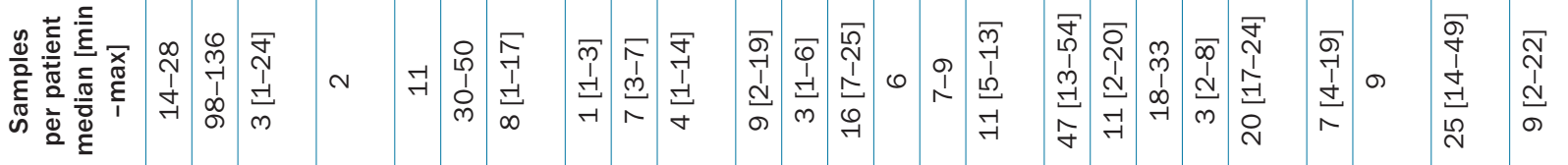

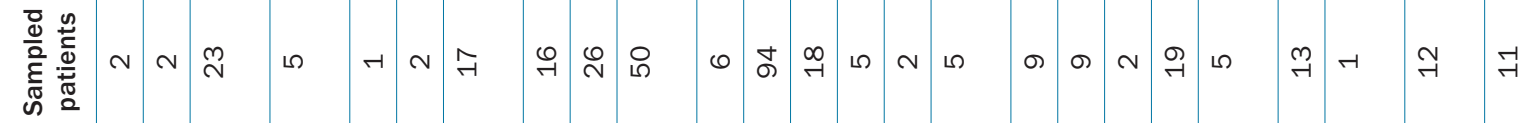

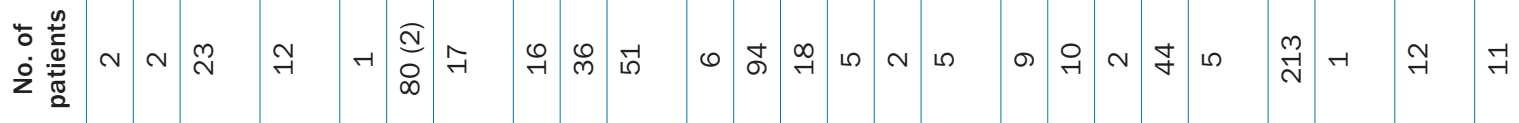

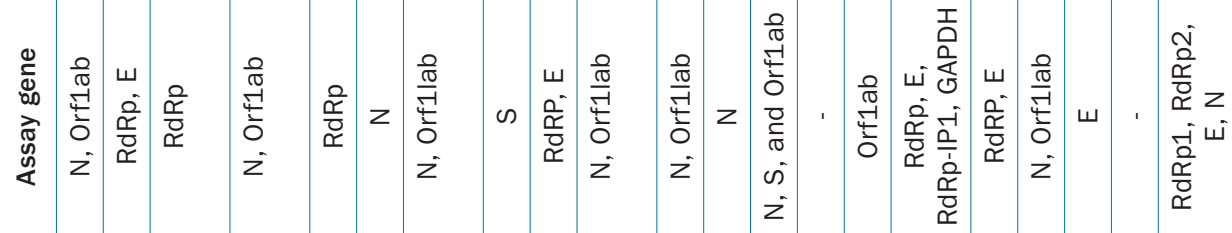

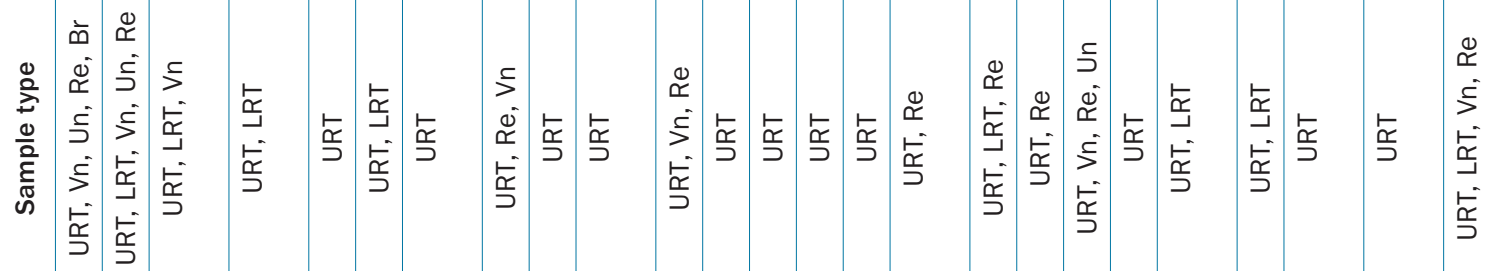

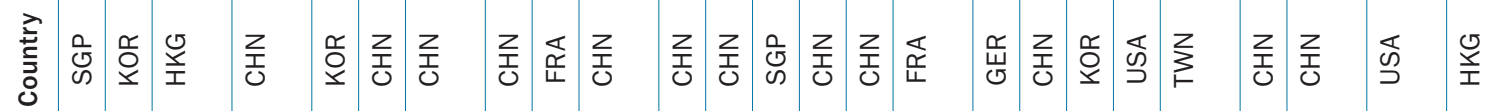

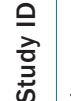

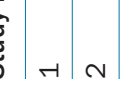




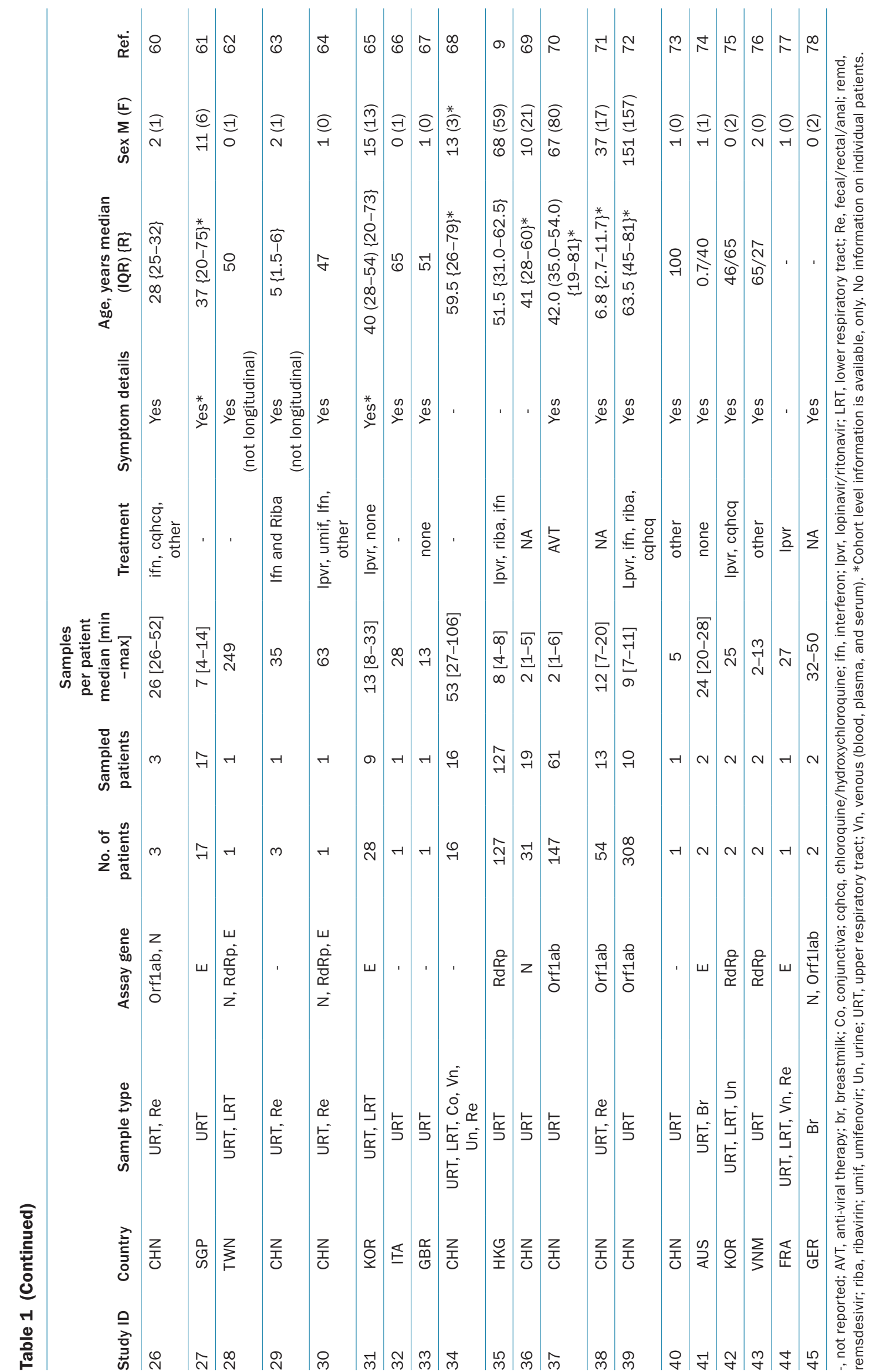


Table 2 Overview extracted variables across different analyses, median [range] (percentage of missing data records)

\begin{tabular}{lccc}
\hline Descriptive (\%missing) & $\begin{array}{c}\text { All data } \\
n=645\end{array}$ & $\begin{array}{c}\text { Cox-PH } \text { full dataset } \\
n=354\end{array}$ & $\begin{array}{c}\text { NLME/ reduced Cox-PH } \\
n=317\end{array}$ \\
\hline Age, years & $46[0.1-100](31.3 \%)$ & $48[0.1-100](0 \%)$ & $46[0.1-100](0 \%)$ \\
\hline Sex, male/female & $217 / 189(37 \%)$ & $215 / 139(0 \%)$ & $182 / 135(0 \%)$ \\
\hline ICU admission, yes/no ${ }^{a}$ & $36 / 371(36.9 \%)$ & $8 / 271(21.2 \%)$ & $8 / 257(16.4 \%)$ \\
\hline Invasive ventilation, yes/no $^{a}$ & $14 / 348(43.9 \%)$ & $9 / 262(23.4 \%)$ & $5 / 247(20.5 \%)$ \\
\hline Death, yes/no & $1 / 455(29.3 \%)$ & $1 / 330(6.5 \%)$ & $1 / 293(7.3 \%)$ \\
\hline Disease severity ${ }^{a}$ & $(12.7 \%)$ & $(0 \%)$ & $(0 \%)$ \\
\hline Asymptomatic & 24 & 19 & 16 \\
\hline Mild & 376 & 258 & 239 \\
\hline Moderate & 79 & 52 & 44 \\
\hline Severe & 84 & 25 & 18 \\
\hline
\end{tabular}

Cox-PH, Cox proportional hazards; ICU, intensive care unit; NLME, nonlinear mixed-effects.

${ }^{\text {a } T h e r e ~ i s ~ d i s c o r d ~ b e t w e e n ~ t h e ~ r e p o r t e d ~ I C U ~ a n d ~ m e c h a n i c a l ~ v e n t i l a t i o n ~ a n d ~ d i s e a s e ~ s e v e r i t y ~ s c o r e ~ d u e ~ t o ~ i n c o m p l e t e ~ r e p o r t i n g ~ i n ~ s o m e ~ p a p e r s . ~ D i s e a s e ~ s e v e r i t y ~}$ was taken from individual reports of disease status in cases where ICU admission and invasive ventilation were not specifically mentioned, and only disease severity was used in the analyses.

ribavirin, and lopinavir/ritonavir plus ribavirin in the Cox- $\mathrm{PH}$ regression analysis showed a trend toward synergy between interferons and ribavirin in the full dataset $(\mathrm{AHR}=6.04, P=0.006$; Figure 3), as well as in the additional analysis taking in quality assessments to account for potential bias: respiratory data limited to drug quality and in data limited to only viral load quality 1 data (Figure S4, Figure S5). Median sampling frequency in the main survival dataset was 1 day but there was a skewed distribution of sampling frequencies with the mean being 1.9 days and $4.8 \%$ of sampling frequencies being $>3$ days. The main analysis was repeated excluding events with sampling frequencies over 3 days to check for potential bias caused by interval censoring, but the main effect sizes were similar (Figure S6).

Covariate relationships and drug effects were explored through NLME modeling with parameter estimates of the model given in Table $\mathbf{S} 4$ along with visual predictive checks and normalized

Pattern of viral shedding in different sites

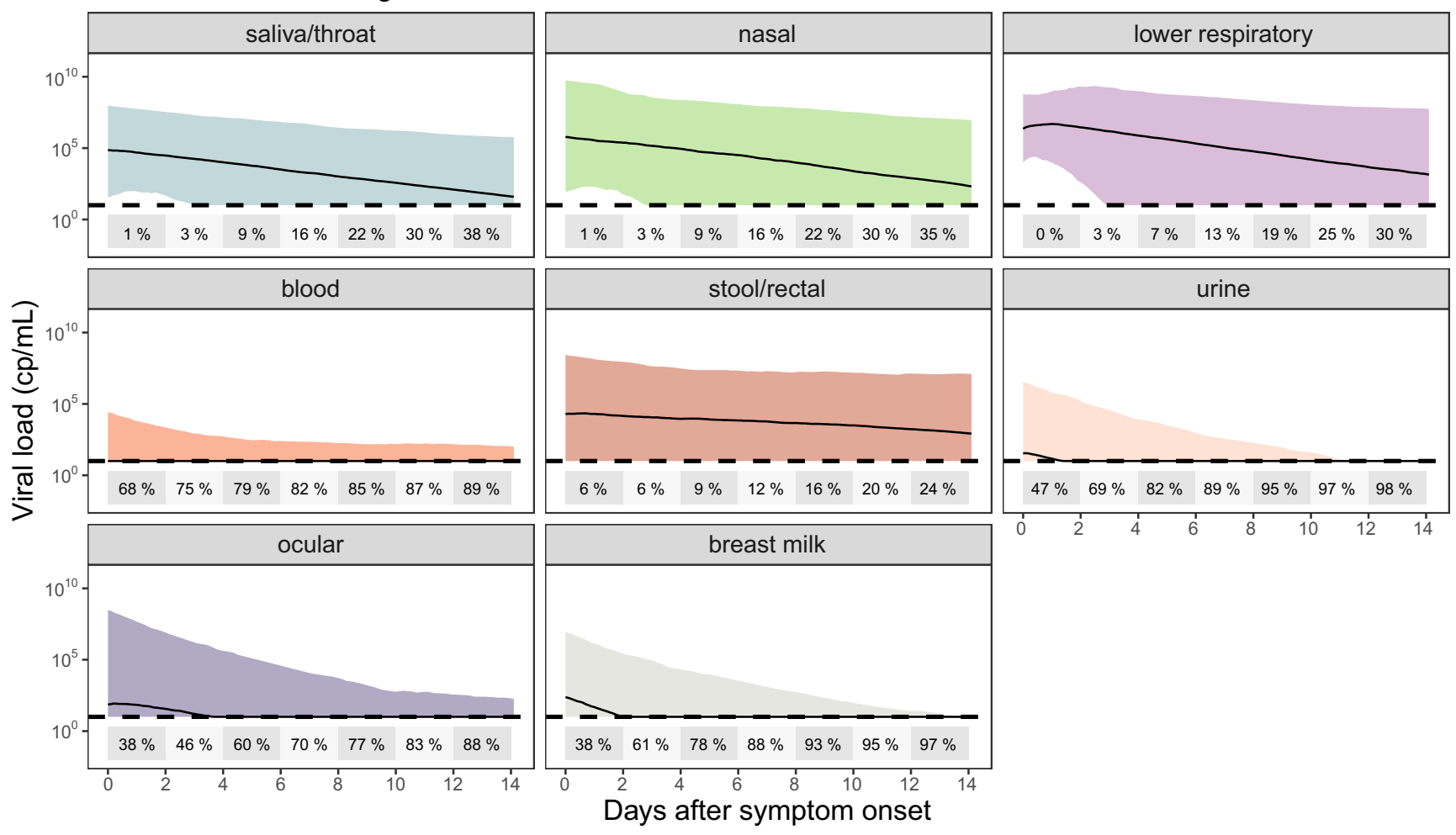

Figure 2 Model-predicted viral load trajectories at each sample site studied. Black lines are the median predictions, with shaded areas representing the $95 \%$ prediction interval. The percentage of samples that are predicted to be below a typical limit of detection (10 copies/mL) are given in two-daily time bins on each plot. [Colour figure can be viewed at wileyonlinelibrary.com] 


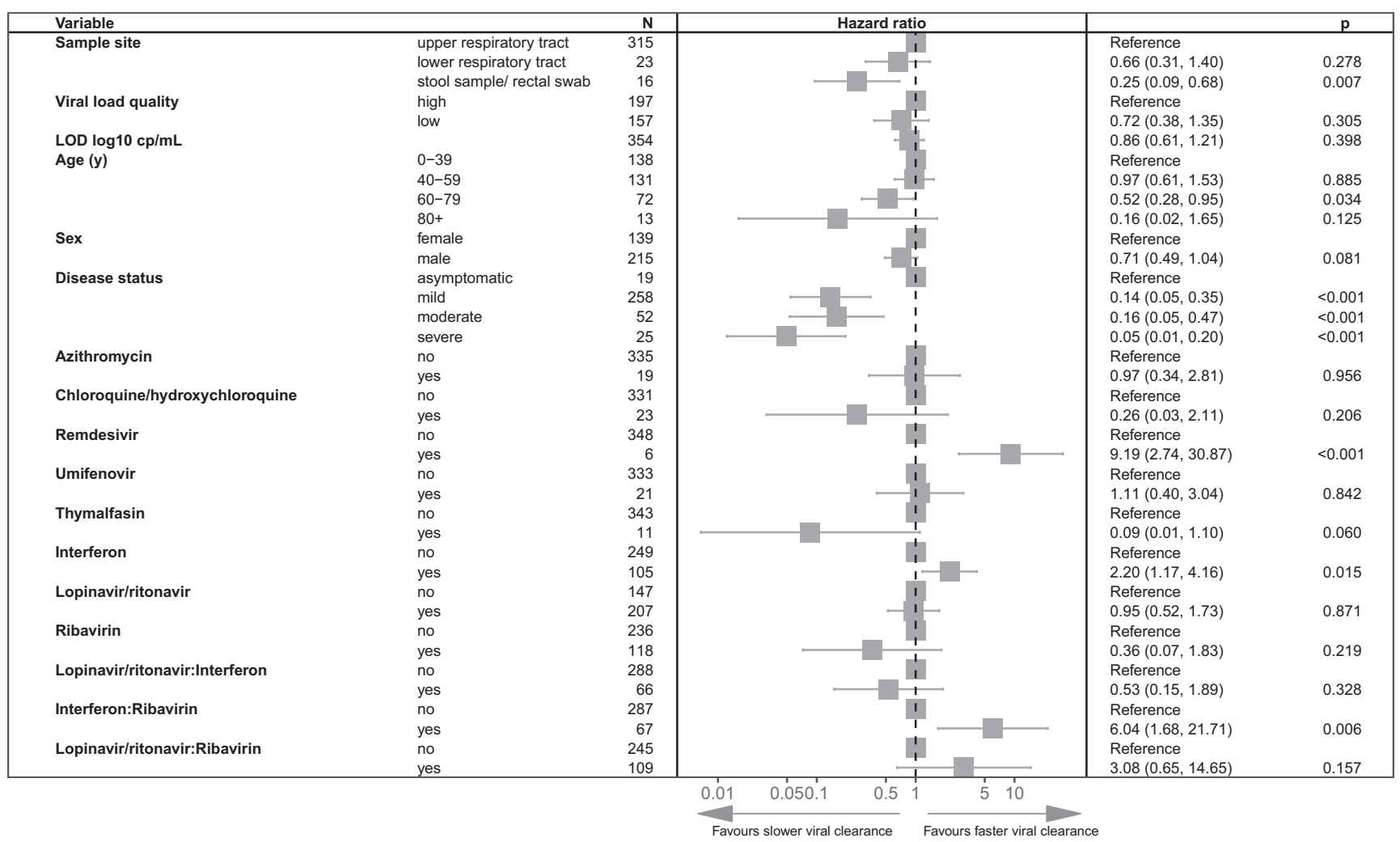

Figure 3 Multivariable Cox proportional hazard results on all drug quality 1 and drug quality 2 data from respiratory and stool/rectal sampling sites. Adjusted hazard ratios exceeding 1 indicate virus being more likely to become undetectable. LOD, limit of detection.

prediction distribution errors in Figure $\mathbf{4}$ and Figure $\mathbf{S} 7$, and visualization of viral AUC, peak viral load, and half-life derived from the final model in Figure S8. Drug effects were estimated to increase $\delta$. Drug regimens containing interferon $(\triangle \mathrm{OFV}=-25.5$, $P<0.001)$, lopinavir/ritonavir $(\Delta \mathrm{OFV}=9.97, P=0.0016)$, and ribavirin $(\Delta \mathrm{OFV}=-22.2, P<0.001)$ each improved model fit and so were taken forward to the final multivariable drug model. The estimated small lopinavir/ritonavir effect on delta, although showing significant model improvement in the likelihood ratio test, did not prove to be robustly detected in the bootstrap analysis, with the interval crossing the value consistent with no drug effect (Table S4). Implementing an additional synergy term, as detected in the Cox-PH model, did not improve the NLME model.

The final model was then used to simulate expected viral trajectories from upper respiratory sampling sites for interferon, and ribavirin monotherapy as well as interferon plus ribavirin combination started at 1, 3, 7, and 10 days post-symptom onset (Figure 5). The sample sizes for hypothetical phase II trials to detect significant differences in viral load vs. no treatment after 7 days of therapy are given in Table $\mathbf{S 5}$.

\section{DISCUSSION}

This systematic review and individual level meta-analysis has identified viral load trajectories from 645 individuals aged from the first month of life to 100 years. Data from all major sampling sites showed, that: following symptom onset in most patients, upper respiratory tract viral load has peaked and is declining, whereas in the lower respiratory tract viral load peaks $2-3$ days after symptom onset; virus is detectable in stool for at least 2 weeks in $75 \%$ of individuals, and virus is detected in low levels in blood, urine, ocular secretions, and breast milk (Figure 2). In addition to simulating the expected trajectory of viral load at each site, we were able to simulate the percentage of samples expected to be below a typical detection limit of 10 copies $/ \mathrm{mL}$ (Figure 2). From this it can be seen that from day 10 post-symptom onset over a quarter of upper respiratory samples have undetectable viral load. This emphasizes the importance of early antiviral therapy, and for phase II trials using viral load as an end point to commence therapy in the first few days of symptom onset in order to reliably differentiate antiviral effects from natural viral decline (Figure 5, Table S5).

Although we followed PRISMA guidelines on individual patient-level meta-analysis methods, registered our review with PROSPERO, and prospectively published our analysis protocol prior to finalizing our search, by including data from case reports, case series, and clinical trials it could be argued that the heterogeneous inclusion criteria of these data may bias the treatment effects we estimated. We therefore repeated the primary analysis on subsets of the data based on sampling site, data quality, and sampling frequency (Figures S4-S6) finding that the main effects were consistent. It should be noted that by far the largest drug quality 1 dataset was the clinical trial from Hung et al. ${ }^{9}$ with 127 patients randomized to either lopinavir/ritonavir vs. lopinavir/ritonavir plus ribavirin plus interferon $\beta$, and our second largest drug quality 1 group was those confirmed to have received no antiviral drugs (67 patients). In total, our NLME dataset contained data on 83 patients receiving interferons, 187 patients receiving lopinavir/ 
quality 1
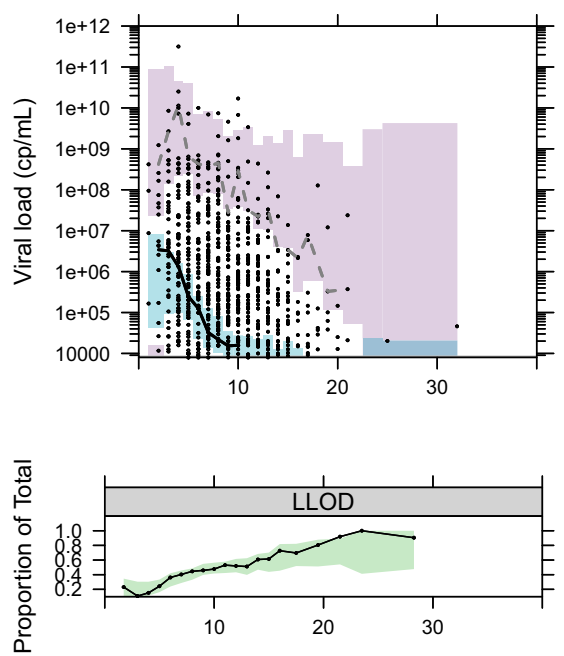

saliva/throat
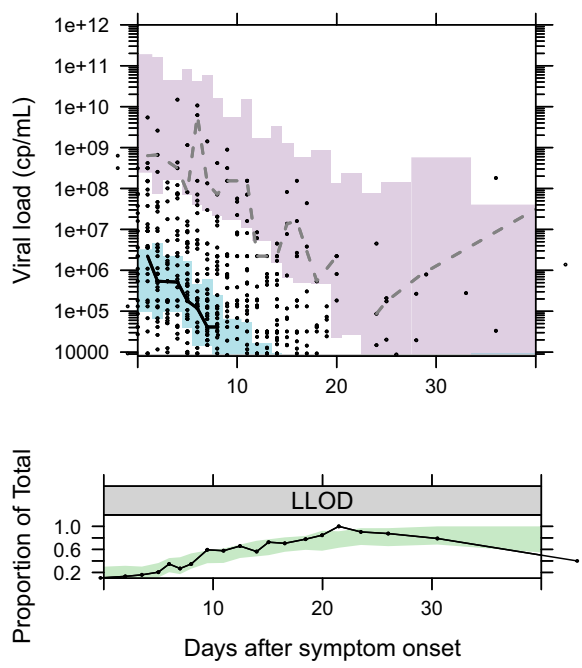

quality 2
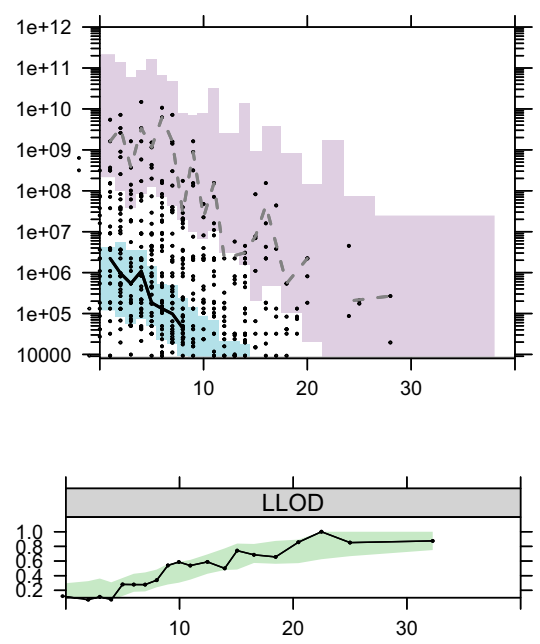

lower respiratory
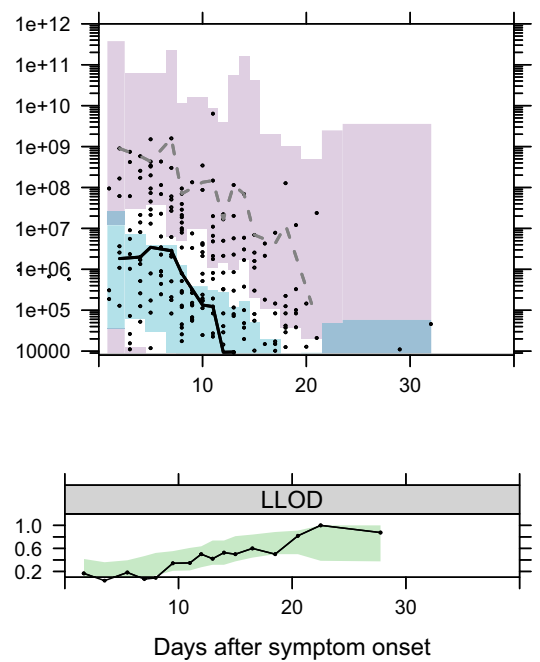

quality 3
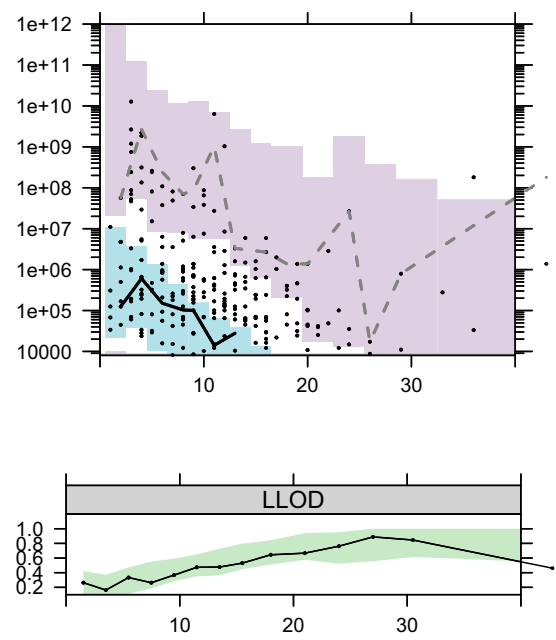

nasal
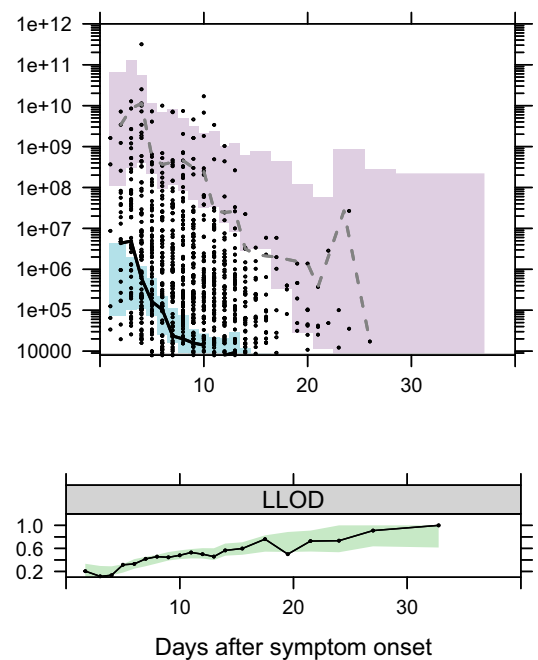

Figure 4 Visual predictive checks for the nonlinear mixed-effects model fitted to viral load data to each sampling site. For each site a plot of model simulations compared with observations is given for both the continuous data (upper) and the fraction of samples below the limit of detection (lower). Black circles are observed viral loads, purple shaded area is the $95 \%$ prediction interval of the simulated 2.5 th and 97.5 th percentile for comparison with the observed 2.5 and 97.5 th percentile (dashed lines). The blue shaded area is the $95 \%$ prediction interval of the 50th percentile to compare with the continuous black line. In the lower plot, the observed proportion of samples below the lower limit of detection (LLOD) are shown as a black line and compared with the 95\% prediction interval of the model predicted proportion of samples below the LLOD (green shaded area). [Colour figure can be viewed at wileyonlinelibrary.com]

ritonavir, and 99 patients receiving ribavirin either alone or in combination (Table S2). Therefore, whereas consistency in results with various subgroup analyses indicate confounding related to heterogeneous data is unlikely to have biased our main findings, the heterogeneity in drug and drug combination studies meant modeling was required to tease out individual drug effects.

A heterogeneous range of antivirals, administered in different combinations, was observed in our data (Table S2) meaning multivariable modeling of time to viral clearance was used to tease out individual drug effects. No antiviral activity was seen for chloroquine/hydroxychloroquine, azithromycin, lopinavir/ritonavir, umifenovir, and thymalfasin. However, remdesivir and interferons were both independently associated with shorter time to viral clearance (Figures 3, S4, S5). Remdesivir did not, however, significantly decrease $\delta$ in the NLME model, but this is likely due to the low number of included patients.

Our most interesting finding is the promising antiviral activity of interferons, possibly due to low endogenous interferon levels induced bySARS-CoV-2. ${ }^{23,24}$ Interferons (alpha and beta) have shown extensive in vitro activity against SARS-CoV-1 and Middle East respiratory syndrome coronavirus (MERS-CoV). ${ }^{25,26}$ However, this has not translated into clinical effectiveness in MERS-CoV, ${ }^{25}$ although results from one trial are still pending. ${ }^{27}$ Although recent data suggests interferon beta may be more potent than alpha against SARS-CoV-2 in vitro, ${ }^{28}$ possibly due to higher selective indices for interferon-beta $1 \mathrm{~b}$, upon finding similar effects of interferon alpha 


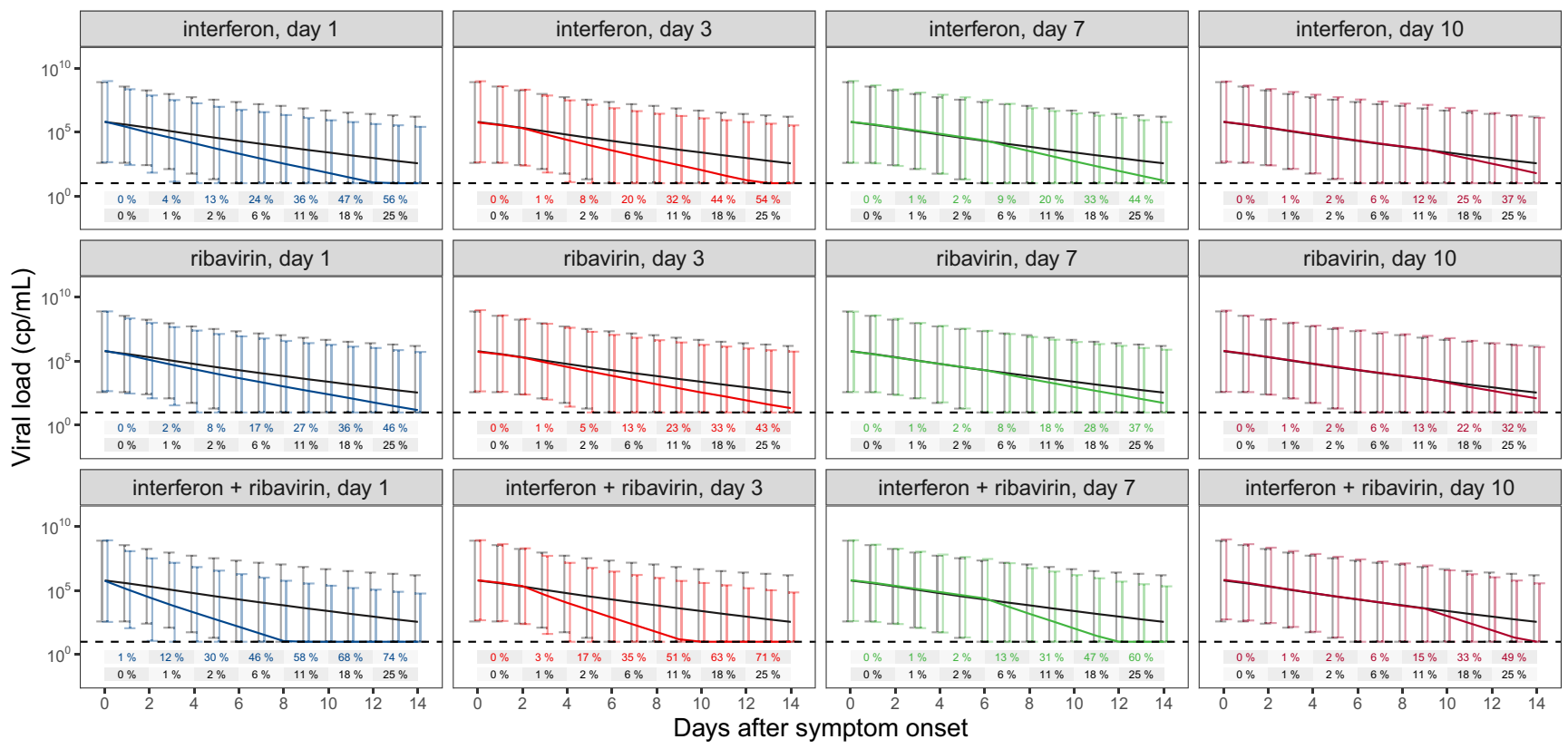

Figure 5 Simulated viral load trajectories. Simulations with a dummy population equally distributed between 50 and 100 years, and equal male/female ratio were performed for each scenario. Drugs were started at day 1 (blue), day 3 (orange), day 7 (green), or day 10 (red) post symptom onset. Mean black line and error bars represent simulations of the dummy population without drug treatment. Colored mean lines and error bars represent the respective drug regimen. Percentage values represent expected proportion of samples below the limit of detection for no drug (black) vs. drug therapy (colored) at each timepoint. [Colour figure can be viewed at wileyonlinelibrary.com]

and beta in our primary analysis (Figure S3), we decided to combine the interferon effect to better explore drug combinations. In the Cox-PH analysis, consistent across data qualities and sampling site combinations, we found either a significant or trend toward significant synergistic activity of interferon plus ribavirin (Figures 3, 4, S4). An extensive body of literature exists to show both interferon alpha and beta are synergistic with ribavirin in vitro against both SARS-CoV-1 and MERS-CoV. ${ }^{25}$ This synergy, however, was not confirmed when tested in the NLME analysis, indicating that the detected synergistic effect from the time to viral clearance analysis might be confounded. Correlations in the timing for start of drug treatment could be one confounder that is corrected for in the NLME approach. The time-dependent analysis from the NLME model suggests an additive effect for interferon and ribavirin, rather than a synergistic effect. Thus, combining interferons with a nucleoside analogue, possibly remdesivir or favipiravir as less toxic alternatives to ribavirin, is a potentially promising combination for viral load suppression. In our secondary analysis, we included interferon plus ribavirin in the NLME model and simulations show that virus should be suppressed 2-3 days faster compared with no treatment (Figure 5). However, it must be noted that recent evidence from the WHO SOLIDARITY trial shows that interferons were associated with a trend to increased mortality ${ }^{29}$ whereas an unpublished press release reports inhaled interferon- $\beta$ to be beneficial. ${ }^{30}$ There is a clear need for a well-designed phase II trial on interferons in early disease to confirm or refute the signal seen in our data.

Another main finding of our work was the limited antiviral effect of lopinavir/ritonavir, in addition to its lack of significant synergistic effect with either ribavirin or interferons. The protease inhibitor lopinavir had a modest but consistent in vitro activity against the major coronaviruses, including SARS-CoV-2, although activity is confined to concentrations at the upper end of the clinically achievable range. ${ }^{1}$ Although lopinavir significantly improved model fit when increasing $\delta$, the bootstrap lower boundary crossed the threshold of no drug effect (Table S4), and our simulations suggest monotherapy studies would require well over 500 participants per arm just to show antiviral activity. As recent phase III trials have now conclusively shown, lopinavir/ritonavir is ineffective in monotherapy. ${ }^{29,31}$ It remains to be seen whether lopinavir/ritonavir may be useful in combinations, however. In SARS-CoV-1, lopinavir/ritonavir plus ribavirin was found to be synergistic in vitro and when initiated immediately upon diagnosis led to a significant decrease in mortality compared with historical controls. ${ }^{32,33}$ Early postexposure prophylaxis against MERS- $\mathrm{CoV}$ in healthcare workers showed that lopinavir/ritonavir plus ribavirin reduced the incidence of infection from $28 \%$ to $0 \%{ }^{34}$ The lopinavir/ritonavir plus ribavirin combination has therefore been the basis for many clinical trials and treatment protocols, but our findings suggest that it may not be as useful in SARS-CoV-2 (Figure 3).

The antiviral effects of remdesivir in vitro are well-established and despite only being able to extract individual patient-level data on six patients, it produced a significantly faster viral clearance in the primary analysis (Figure 3). Despite in some cases showing promising in vitro activity, we did not find significant antiviral effects of azithromycin, chloroquine/hydroxychloroquine, thymalfasin, or umifenovir. In the case of hydroxychloroquine and azithromycin, the raw viral load data from the heavily criticized study by Gautret $e t$ al. ${ }^{35}$ was included, but contrary to the original analysis we found no clinical antiviral activity of either drug and, in the case of hydroxychloroquine, a trend toward slower viral clearance. The reason for this difference in interpretation appears to stem from using time since symptom onset as opposed to time 
since starting drug and with untreated patients being monitored from an earlier day post-symptom onset. This example highlights the necessity of accounting for the time course of the infection when analyzing viral loads.

In our secondary NLME analysis, the simplified target cell limited model provided a good fit to data from each sampling site. In many cases, this approximated a mono-exponential decay, but in others, particularly in lower respiratory tract, there was a pronounced peak in the first days following symptom onset. The model was stable with high interindividual variability on $\mathrm{V}_{0}$ and $\beta$, reflecting the fact that relative changes in these parameters lead to the initial part of the curve either rising then falling (in situations when $V_{0} \approx-\beta$ ) or approximately monoexponentially declining (when $V(0)>>-\beta$ ). In addition, we found the model to be less sensitive to changes in $\gamma$, meaning it can take a wide range of values with little influence on model fit, hence we did not estimate an interindividual variability term on it. Increasing age was associated with significantly slower $\delta$, and there was a small effect of male sex also being associated with slower $\delta$ (Table S4). The age effect translates to a 5 -year-old having a viral decay terminal half-life of 1.0 day, a 47 -year-old (median age in our population) 1.18 days, and a 90 -year-old 1.24 days. Hence, a child has an almost $15 \%$ faster viral clearance than a middle-aged adult, and almost $20 \%$ faster than an elderly person.

In contrast to authors who have estimated parameters for more mechanistic models, ${ }^{36}$ we estimated all drug effects to increase $\delta$, which implies a mode of action relating to inhibition of viral replication or stimulation of viral clearance mechanisms. Although for most of the drugs studied this may be reasonable, entry inhibitors may be more appropriately described by inhibition of $\gamma$, which may not be statistically identifiable with the data possible to collect in the clinical setting. Despite this potential limitation, we found similar agents (combinations including interferons and ribavirin) to those identified in the primary analysis of time to viral clearance.

The major limitation of our work is the lack of clinical trial data and lack of data on potentially important repurposing agents, such as favipiravir and nitazoxanide, and that only one of the authors of a major clinical trial agreed to share their data.9 Through applying quality assessment criteria on drug history and assay reporting, prespecifying our analysis in our protocol and PROSPERO registration before undertaking Cox-PH and NLME modeling we aimed to reduce possible bias in the heterogenous data available. Although we were able to extract a limited common demographics set, particularly in the high-quality data subset (age, sex, disease severity, and antiviral drug histories), our data may be limited by other nonantiviral medications that were not fully reported in the included papers. Furthermore, as many of our included papers were on patients with mild or no symptoms and only contained data on one patient reported to have died, we were unable to study associations of viral load and mortality. Viral load measured by PCR is not necessarily infectious virus, and recently it has been shown that only in samples above $10^{7}$ copies $/ \mathrm{mL}$ can SARS-CoV-2 be cultured. ${ }^{39}$ Therefore, our data should preferably be used to study viral trajectories in relation to antiviral therapy rather than to infer probability of transmission.

The detection of viable virus might be overcome through whole genome sequencing and the detection of subgenomic RNA. This has, however, only been conducted in a single study, included in our review. Woelfel $e$ a l. $^{37}$ showed through E gene subgenomic RNA quantification and relating it to the entire virus genome RNA, that presence of subgenomic RNA fragments can be a hint for active viral replication and thus active infection. More recent studies by Alexandersen et al..$^{38}$ and van Kampen et al. ${ }^{39}$ however, detected subgenomic RNA up to 22 days after onset of symptoms. It is postulated this was related to subgenomic RNA being rather stable and associated with cellular membranes and thus detection of subgenomic RNAs in clinical samples does not necessarily indicate viral activity. Future controlled studies of subgenomic RNA levels in patients on and off antiviral therapies are urgently required to better understand this potential biomarker of drug effect.

In conclusion, this individual patient level meta-analysis has yielded useful insights into SARS-CoV-2 viral dynamics. A modelbased description of viral trajectories in different sampling sites has been elucidated, and we have found covariates, such as increasing age, disease severity, and male sex to be associated with slower viral clearance. Our review firmly establishes a role for early viral suppression in the management of SARS-CoV-2 and an important signal as to the possible benefits of interferons as a component of antiviral therapy has been found. It has been shown that viral dynamic models such as ours can increase the power to detect drug effects due to their utilization of serial measures ${ }^{40}$ and our model should be useful to others in both the design and analysis of future phase II trials, hence the model code and raw data from this analysis are made available.

\section{Supporting Information}

Supplementary information accompanies this paper on the Clinical Pharmacology \& Therapeutics website (www.cpt-journal.com).

\section{FUNDING}

No specific funding was available for this work. Support at the institution level came from the National Institute for Health Research Biomedical Research Centre at Great Ormond Street Hospital for Children NHS Foundation Trust and University College London, and J.F.S. and F.K. were supported by United Kingdom Medical Research Council (MRC) Fellowships (grants M008665 and P014534). J.B. receives funding from the NIHR UCL/UCLH Biomedical Research Centre. J.P. is supported by a Rosetrees Trust PhD fellowship (M876). F.T.B. is supported by a Wellcome Trust Collaborative Award (203268) to J.B.

\section{CONFLICT OF INTEREST}

The authors declared no competing interests for this work. As an Associate Editor of Clinical Pharmacology and Therapeutics, Joseph F. Standing was not involved in the review or decision process for this paper.

\section{CODE AVAILABILITY}

Model code is available at: https:/github.com/ucl-pharmacometrics/ SARS-CoV-2-viral-dynamic-meta-analysis.

\section{DATA AVAILABILITY STATEMENT}

The final dataset is available at: https://github.com/ucl-pharmacome trics/SARS-CoV-2-viral-dynamic-meta-analysis.

(c) 2021 The Authors. Clinical Pharmacology \& Therapeutics published by Wiley Periodicals LLC on behalf of American Society for Clinical Pharmacology and Therapeutics

This is an open access article under the terms of the Creative Commons Attribution License, which permits use, distribution and reproduction in any medium, provided the original work is properly cited. 
1. Arshad, U. et al. Prioritization of anti-SARS-Cov-2 drug repurposing opportunities based on plasma and target site concentrations derived from their established human pharmacokinetics. Clin. Pharmacol. Ther. 108, 775-790 (2020).

2. To, K.K. et al. Temporal profiles of viral load in posterior oropharyngeal saliva samples and serum antibody responses during infection by SARS-CoV-2: an observational cohort study. Lancet Infect. Dis. 20, 565-574 (2020).

3. He, X. et al. Temporal dynamics in viral shedding and transmissibility of COVID-19. Nat. Med. 26, 672-675 (2020).

4. Rajpal, A., Rahimi, L. \& Ismail-Beigi, F. Factors leading to high morbidity and mortality of COVID-19 in patients with type 2 diabetes. J. Diabetes 12, 895-908 (2020).

5. Fishman, J.A. \& Grossi, P.A. Novel Coronavirus-19 (COVID-19) in the immunocompromised transplant recipient: \#Flatteningthecurve. Am. J. Transplant. 20, 1765-1767 (2020).

6. Zheng, S. et al. Viral load dynamics and disease severity in patients infected with SARS-CoV-2 in Zhejiang Province, China, January-March 2020: retrospective cohort study. BMJ 369, m1443 (2020).

7. Kamal, M.A. et al. A drug-disease model describing the effect of oseltamivir neuraminidase inhibition on influenza virus progression. Antimicrob. Agents Chemother. 59, 5388-5395 (2015).

8. Kim, J.Y. et al. Viral load kinetics of SARS-CoV-2 infection in first two patients in Korea. J. Korean Med. Sci. 35, e86 (2020).

9. Hung, I.F. et al. Triple combination of interferon beta-1b, lopinavirritonavir, and ribavirin in the treatment of patients admitted to hospital with COVID-19: an open-label, randomised, phase 2 trial. Lancet 395, 1695-1704 (2020).

10. Recovery trial. Statement from the chief investigators of the randomised evaluation of COVid-19 thERapY (RECOVERY) Trial on lopinavir-ritonavir. 2020 <https://www.recoverytrial.net/files/ lopinavir-ritonavir-recovery-statement-29062020_final.pdf>. Accessed June 29, 2020.

11. Stewart, L.A. et al. Preferred reporting items for systematic review and meta-analyses of individual participant data: the PRISMA-IPD Statement. JAMA 313, 1657-1665 (2015).

12. Rohatgi, A. Webplotdigitizer: Version 4.2. 2019 [cited] <https:// automeris.io/WebPlotDigitizer>.

13. Vogels, C.B.F. et al. Analytical sensitivity and efficiency comparisons of SARS-COV-2 qRT-PCR primer-probe sets. medRxiv 2020.2003.2030.20048108. (2020)

14. Chu, D.K.W. et al. Molecular diagnosis of a novel coronavirus (2019-nCoV) causing an outbreak of pneumonia. Clin. Chem. 66, 549-555 (2020).

15. Grambsch, P.M. \& Therneau, T.M. Proportional hazards tests and diagnostics based on weighted residuals. Biometrika 81, 515526 (1994).

16. R Core Team. A Language and Environment for Statistical Computing (version 3.6.3) (R Foundation for Statistical Computing, Vienna, Austria, 2019).

17. Baccam, P., Beauchemin, C., Macken, C.A., Hayden, F.G. \& Perelson, A.S. Kinetics of influenza A virus infection in humans. J. Virol. 80, 7590-7599 (2006).

18. Anguelova, M., Karlsson, J. \& Jirstrand, M. Minimal output sets for identifiability. Math. Biosci. 239, 139-153 (2012).

19. Kim, K.S. et al. Modelling SARS-CoV-2 dynamics: implications for therapy. medRxiv 2020.2003.2023.20040493 (2020)

20. Beal, S., Sheiner, L.B., Boeckmann, R. \& Bauer, R.J. NONMEM 7.4 users guides. (1989-2018) [cited] <https://nonmem.iconplc.com/ nonmem743/guides $>$.

21. Nguyen, T.H. et al. Model evaluation of continuous data pharmacometric models: metrics and graphics. CPT Pharmacometrics Syst. Pharmacol. 6, 87-109 (2017).

22. Beal, S.L. Ways to fit a PK model with some data below the quantification limit. J. Pharmacokinet. Pharmacodyn. 28, 481-504 (2001).

23. Blanco-Melo, D. et al. Imbalanced host response to SARS-CoV-2 drives development of COVID-19. Cell 181, 1036-1045 e1039 (2020).

24. Chu, H. et al. Comparative replication and immune activation profiles of SARS-CoV-2 and SARS-CoV in human lungs: an ex vivo study with implications for the pathogenesis of COVID-19. Clin. Infect. Dis. 71, 1400-1409.(2020).

25. Zeitlinger, M. et al. Pharmacokinetics/pharmacodynamics of antiviral agents used to treat SARS-CoV-2 and their potential interaction with drugs and other supportive measures: a comprehensive review by the PK/PD of Anti-Infectives Study Group of the European Society of Antimicrobial Agents. Clin. Pharmacokinet. 59, 1195-1216 (2020).

26. Sheahan, T.P. et al. Comparative therapeutic efficacy of remdesivir and combination lopinavir, ritonavir, and interferon beta against MERS-CoV. Nat. Commun. 11, 222 (2020).

27. Arabi, Y.M. et al. Treatment of middle east respiratory syndrome with a combination of lopinavir-ritonavir and interferon-beta1b (MIRACLE trial): study protocol for a randomized controlled trial. Trials 19, 81 (2018).

28. Yuan, S. et al. Broad-spectrum host-based antivirals targeting the interferon and lipogenesis pathways as potential treatment options for the pandemic coronavirus disease 2019 (COVID-19). Viruses 12, 628 (2020)

29. Pan, H. et al. Repurposed antiviral drugs for COVID-19 -interim WHO SOLIDARITY trial results. medRxiv 2020.2010.2015.20209817. (2020).

30. Synairgen plc. Interim results for the six months ended 30 June 2020. 2020 <https://www.synairgen.com/wp-content/uploa ds/2020/09/200929-Synairgen-Interim-Results-final.pdf>.

31. Recovery Collaborative Group. Lopinavir-ritonavir in patients admitted to hospital with COVID-19 (RECOVERY): a randomised, controlled, open-label, platform trial. Lancet 396, 1345-1352 (2020).

32. Chu, C.M. et al. Role of lopinavir/ritonavir in the treatment of SARS: initial virological and clinical findings. Thorax 59, 252-256 (2004).

33. Chan, K.S. et al. Treatment of severe acute respiratory syndrome with lopinavir/ritonavir: a multicentre retrospective matched cohort study. Hong Kong Med. J. 9, 399-406 (2003).

34. Park, S.Y. et al. Post-exposure prophylaxis for Middle East respiratory syndrome in healthcare workers. J. Hosp. Infect. 101, 42-46 (2019).

35. Gautret, P. et al. Hydroxychloroquine and azithromycin as a treatment of COVID-19: results of an open-label nonrandomized clinical trial. Int. J. Antimicrob. Agents 56, 105949 (2020).

36. Goncalves, A. et al. Timing of antiviral treatment initiation is critical to reduce SARS-CoV-2 viral load. CPT Pharmacometrics Syst. Pharmacol. 9, 509-514 (2020).

37. Wolfel, R. et al. Virological assessment of hospitalized patients with COVID-2019. Nature 581, 465-469 (2020).

38. Alexandersen, S., Chamings, A. \& Bhatta, T.R. SARSCoV-2 genomic and subgenomic RNAs in diagnostic samples are not an indicator of active replication. medRxiv 2020.2006.2001.20119750 (2020).

39. van Kampen, J.J.A. et al. Duration and key determinants of infectious virus shedding in hospitalized patients with coronavirus disease-2019 (COVID-19). Nat. Commun. 12, 267 (2021). https:// doi.org/10.1038/s41467-020-20568-4

40. Laouenan, C., Guedj, J. \& Mentre, F. Clinical trial simulation to evaluate power to compare the antiviral effectiveness of two hepatitis $C$ protease inhibitors using nonlinear mixed effect models: a viral kinetic approach. BMC Med. Res. Methodol. 13, 60 (2013).

41. Kam, K.Q. et al. A well infant with coronavirus disease 2019 with high viral load. Clin. Infect. Dis. 71, 847-849 (2020).

42. Liu, Y. et al. Clinical and biochemical indexes from 2019-nCoV infected patients linked to viral loads and lung injury. Sci. China Life Sci. 63, 364-374 (2020).

43. Lim, J. et al. Case of the index patient who caused tertiary transmission of COVID-19 infection in Korea: the application of lopinavir/ritonavir for the treatment of COVID-19 infected pneumonia monitored by quantitative RT-PCR. J. Korean Med. Sci. 35, e79 (2020).

44. Pan, Y., Zhang, D., Yang, P., Poon, L.L.M. \& Wang, Q. Viral load of SARS-CoV-2 in clinical samples. Lancet Infect. Dis. 20, 411-412 (2020). 
45. Zhang, W. et al. Molecular and serological investigation of 2019nCoV infected patients: implication of multiple shedding routes. Emerg. Microbes Infect. 9, 386-389 (2020).

46. $\mathrm{Xu}, \mathrm{T}$. et al. Clinical features and dynamics of viral load in imported and non-imported patients with COVID-19. Int. J. Infect. Dis. 94, 68-71 (2020).

47. Chen, W. et al. Detectable 2019-nCoV viral RNA in blood is a strong indicator for the further clinical severity. Emerg. Microbes Infect. 9, 469-473 (2020).

48. Young, B.E. et al. Epidemiologic features and clinical course of patients infected with SARS-CoV-2 in Singapore. JAMA $\mathbf{3 2 3}$, 1488-1494 (2020).

49. Shen, C. et al. Treatment of 5 critically ill patients with COVID-19 with convalescent plasma. JAMA 323, 1582-1589 (2020).

50. Wan, R., Mao, Z.Q., He, L.Y., Hu, Y.C. \& Wei, C. Evidence from two cases of asymptomatic infection with SARS-CoV-2: are 14 days of isolation sufficient? Int. J. Infect. Dis. 95, 174-175 (2020).

51. Lescure, F.X. et al. Clinical and virological data of the first cases of COVID-19 in Europe: a case series. Lancet Infect Dis. 20, 697706 (2020).

52. Xu, Y. et al. Characteristics of pediatric SARS-CoV-2 infection and potential evidence for persistent fecal viral shedding. Nat. Med. 26, 502-505 (2020).

53. Han, M.S. et al. Sequential analysis of viral load in a neonate and her mother infected with SARS-CoV-2. Clin. Infect. Dis. 71, 22362239 (2020).

54. Wyllie, A.L. et al. Saliva is more sensitive for SARS-CoV-2 detection in COVID-19 patients than nasopharyngeal swabs. medRxiv 2020.2004.2016.20067835 (2020)

55. Cheng, C.Y. et al. Lopinavir/ritonavir did not shorten the duration of SARS CoV-2 shedding in patients with mild pneumonia in Taiwan. J. Microbiol. Immunol. Infect. 53, 488-492 (2020).

56. Yang, Y. et al. Evaluating the accuracy of different respiratory specimens in the laboratory diagnosis and monitoring the viral shedding of 2019-nCoV infections. medRxiv 2020.2002.2011.20021493 (2020)

57. Yang, J.R. et al. Persistent viral RNA positivity during the recovery period of a patient with SARS-CoV-2 infection. J. Med. Virol. 92, 1681-1683 (2020).

58. COVID Investigation Team. Clinical and virologic characteristics of the first 12 patients with coronavirus disease 2019 (COVID-19) in the United States. Nat. Med. 26, 861-868 (2020).

59. Lui, G. et al. Viral dynamics of SARS-CoV-2 across a spectrum of disease severity in COVID-19. J. Infect. 81, 318-356 (2020).

60. $\mathrm{Hu}, \mathrm{Y}$. et al. A report of three COVID-19 cases with prolonged viral RNA detection in anal swabs. Clin. Microbiol. Infect. 26, 786-787 (2020).

61. Seah, I.Y.J. et al. Assessing viral shedding and infectivity of tears in coronavirus disease 2019 (COVID-19) patients. Ophthalmology 127, 977-979 (2020).
62. Liu, W.D. et al. Prolonged virus shedding even after seroconversion in a patient with COVID-19. J. Infect. 81, 318-356 (2020).

63. Xing, Y.H. et al. Prolonged viral shedding in feces of pediatric patients with coronavirus disease 2019. J. Microbiol. Immunol. Infect. 53, 473-480 (2020).

64. Qian, G.Q. et al. Duration of SARS-CoV-2 viral shedding during COVID-19 infection. Infect. Dis. (Lond). 52, 511-512 (2020).

65. Kim, E.S. et al. Clinical course and outcomes of patients with severe acute respiratory syndrome coronavirus 2 infection: a preliminary report of the first 28 patients from the Korean Cohort Study on COVID-19. J. Korean Med. Sci. 35, e142 (2020).

66. Colavita, F. et al. SARS-CoV-2 Isolation from ocular secretions of a patient with COVID-19 in Italy with prolonged viral RNA detection. Ann. Intern. Med. 173, 242-243 (2020).

67. Hill, K.J. et al. The index case of SARS-CoV-2 in Scotland. J. Infect. 81, 147-178 (2020).

68. Huang, Y. et al. SARS-CoV-2 viral load in clinical samples from critically ill patients. Am. J. Respir. Crit. Care Med. 201, 14351438 (2020).

69. Zhou, R. et al. Viral dynamics in asymptomatic patients with COVID-19. Int. J. Infect. Dis. 96, 288-290 (2020).

70. Qi, L. et al. Factors associated with the duration of viral shedding in adults with COVID-19 outside of Wuhan, China: a retrospective cohort study. Int. J. Infect. Dis. 96, 531-537 (2020).

71. Yuan, C. et al. Viral loads in throat and anal swabs in children infected with SARS-CoV-2. Emerg. Microbes Infect. 9, 1233-1237 (2020).

72. Huang, J.T. et al. Chronological changes of viral shedding in adult inpatients with COVID-19 in Wuhan, China. Clin. Infect. Dis. $\mathbf{7 1}$ 2158-2166 (2020).

73. Kong, Y. et al. Successful treatment of a centenarian with coronavirus disease 2019 (COVID-19) using convalescent plasma. Transfus Apher. Sci. 59, 102820 (2020).

74. Tam, P.C.K. et al. Detectable severe acute respiratory syndrome coronavirus 2 (SARS-CoV-2) in human breast milk of a mildly symptomatic patient with coronavirus disease 2019 (COVID-19). Clin. Infect. Dis. 72, 128-130 (2020).

75. Yoon, J.G. et al. Clinical significance of a high SARS-CoV-2 viral load in the saliva. J. Korean Med. Sci. 35, e195 (2020).

76. Phan, L.T. et al. Clinical features, isolation, and complete genome sequence of severe acute respiratory syndrome coronavirus 2 from the first two patients in Vietnam. J. Med. Virol. 92, 22092215 (2020).

77. Klement-Frutos, E. et al. Early administration of ritonavir-boosted Iopinavir could prevent severe COVID-19. J. Infect. 82, 159-198 (2020).

78. Gross, R. et al. Detection of SARS-CoV-2 in human breastmilk. Lancet 395, 1757-1758 (2020). 